\title{
Modulation of Cardiac Metabolism in Heart
}

\section{Failure}

\section{Giuseppe MC Rosano ${ }^{1}$, Andrew JS Coats ${ }^{2}$}

1. Centre for Clinical \& Basic Research IRCCS San Raffaele Pisana, via della Pisana, 235, 00163 Rome, Italy

2. President-Elect, Heart Failure Association of the ESC, IRCCS, San Raffaele, Pisana, Rome

\section{Corresponding author:}

Andrew JS Coats,

IRCCS, San Raffaele, Pisana, Rome, Italy.

Email: ajscoats@aol.com

\begin{abstract}
Heart failure is associated with altered cardiac metabolism, in part, due to maladaptive mechanisms, in part secondary to comorbidities such as diabetes and ischaemic heart disease. The metabolic derangements taking place in heart failure are not limited to the cardiac myocytes, but extend to skeletal muscles and the vasculature causing changes that contribute to the worsening of exercise capacity. Modulation of cardiac metabolism with partial inhibition of free fatty acid oxidation has been shown to be beneficial in patients with heart failure. At the present, the bulk of evidence for this class of drugs comes from Trimetazidine. Newer compounds partially inhibiting free fatty acid oxidation or facilitating the electron transport on the mitochondrial cristae are in early phase of their clinical development.
\end{abstract}

Keywords: $\quad$ heart failure; metabolism; trimetazidine; mitochondria

Citation: $\quad$ Rosano GMC, Coats AJS. Modulation of Cardiac Metabolism in Heart Failure. International Cardiovascular Forum Journal. 2019;17:22-25. DOI: 10.17987/icfj.v17i0.597

\section{Introduction}

Heart failure is associated with altered cardiac metabolism.[1] The changes in cardiac metabolism are, in part, due to maladaptive mechanisms, in part secondary to comorbidities such as diabetes and ischaemic heart disease. As a consequence, HF should be regarded as a systemic and multi-organ syndrome with metabolic failure, and the failing heart can be regarded as "an engine out of fuel".[2] The metabolic derangements taking place in heart failure are not limited to the cardiac myocytes, but extend to skeletal muscles and the vasculature causing changes that contribute to the worsening of exercise capacity [fatigue, muscle weakness, exercise limitation] and to disease progression.[1] Furthermore, with the heart and skeletal muscle being less metabolically efficient when these metabolic changes occur, patients will expend more energy for any given level of exercise.

\section{Cardiac metabolism in the normal and in the failing heart}

At rest cardiac myocytes derive their main source of energy from free fatty acid oxidation, with up to $80 \%$ of high energy phosphates being produced through this reaction. The remaining energy is produced by glucose oxidation. Excess glucose taken up by the myocytes is stored in the form of glycogen in order to be used in states of increased metabolic demand. Glucose utilisation is $20-30 \%$ more metabolically efficient compared to free fatty acid oxidation in the production of high energy phosphates.[3]
The heart at rest uses a less efficient metabolic pathway in order to store more efficient fuel for states of increased metabolic demands such as in the presence of increased heart rate and blood pressure or the need for a higher cardiac output. An increase in glucose and lactate uptake and utilisation without significant changes in free fatty acid (FFA) metabolism occurs during low to moderate intensity exercise.[4-6]

Therefore, the myocardium adapts its substrate utilisation according to the metabolic demands at the time; at rest this is only $15-20 \%$ of its maximal oxidative capacity.[4] When the myocardial metabolic requirements exceed the limit of its metabolic reserve, the myocardium reaches its anaerobic threshold. When glucose is used as a substrate the anaerobic threshold is higher as compared to when fatty acids are used.

Maladaptive myocardial energetics play a key role in the pathophysiology and progression of heart failure. A ketosisprone state is observed in patients with heart failure in whom blood ketone bodies and free fatty acid levels are higher during fasting, and remain high after glucose infusion.[7] In patients with heart failure the plasma levels of ketone bodies are related to the degree of neurohormonal activation and the severity of cardiac dysfunction.[8] One cause for these maladaptive changes occurring in patients with heart failure 
is cardiac insulin resistance that develops early in the natural history of heart failure and that reduces the myocardial utilisation of glucose and favours the utilisation of free fatty acids. These changes in cardiac metabolism reduce the cardiac metabolic efficiency thereby reducing the production of high-energy phosphates. Cardiac metabolic derangements occurring in heart failure favour its progression, by reducing cardiac and skeletal muscle efficiency and thereby further reducing functional capacity.

The reduced production of high energy phosphates limits their availability and reserves and impairs myocardial contractility and relaxation.[9] This cardiac and skeletal muscle "metabolic remodelling", with the consequent reduction in the production of high energy phosphates, leads to a progressive worsening of both diastolic and systolic function and muscle contractile strength, and ultimately can lead to muscle mass loss and to the progression of left ventricular remodelling.[10] As heart failure progresses, the presence of a compensatory hyperadrenergic state results in an elevated level of free fatty acids in the serum that leads to the storage of free fatty acids as intra-myocardial triglycerides that causes both lipo-toxicity and worsening of heart failure.

At the cellular level, the altered utilisation of glucose and free fatty acids induces maladaptive cellular changes with decreased mitochondrial cristae, decrease in cellular size and myofibrillar content and reduction in actino-myosin coupling. Therefore, interventions aimed at optimising cardiac and skeletal muscle metabolism, and increasing high energy phosphate production may represent a complementary approach to the treatment of heart failure.[11]

\section{Treatment of HF with metabolic agents}

As mentioned in the section on inotropes, agents that increase cardiac energy consumption have negative long-term prognostic effects that may be related to the exhaustion of high-energy phosphates leading to altered handling of $\mathrm{Ca}+2$ and favouring arrhythmias. Several agents have been suggested to improve cardiac metabolism, to date, of these agents, only trimetazidine and perhexiline have been approved for human use. Trimetazidine and perhexiline are approved for the treatment of angina pectoris and myocardial ischaemia. Both drugs partially inhibit myocardial fatty acid oxidation, improve myocardial energy production and improve regional and global myocardial function.[12] Trimetazidine is available worldwide (except USA where its dossier has never been submitted), while perhexiline is available in very few countries, perhaps because of its dubious safety profile.[13-14] Both agents inhibits FFA oxidation directly by blocking 3-ketoacyl-coenzyme A thiolase (3-KAT) and shift myocardial energy production from free fatty acids to glucose. Studies have demonstrated the benefits of these compounds on myocardial function in patients with heart failure.

Trimetazidine is widely used in the treatment of stable angina pectoris and has a relatively significant amount of evidence for use in the treatment of heart failure. The effects of trimetazidine in heart failure are related to the improved myocardial production of high energy phosphates.[11]

\section{Trimetazidine}

Metabolic modulation of cardiac metabolism with trimetazidine is associated with an improvement of the cardiac phospho-creatine/ ATP ratio by $33 \%$. This effect translates into a parallel improvement of left ventricular function and with a reduction in the whole-body energy expenditure. Trimetazidine (TMZ) has consistently been shown to improve left ventricular ejection fraction, to reduce the New York Heart Association (NYHA) class, to decrease the rate of hospitalisations and to reduce the levels of brain natriuretic peptide (BNP) in patients with heart failure and reduced ejection fraction (HFrEF).[15-26] A number of these studies have also suggested that trimetazidine is effective in reducing hospitalisations and allcause mortality in patients with HFrEF.

Studies have shown that trimetazidine improves cardiac function, exercise capacity and prognosis in patients with heart failure. Randomised clinical trials demonstrated that TMZ, added to optimal heart failure therapy, improves left ventricular end-systolic volume and ejection fraction and functional class in patients with heart failure. A multicentre cohort study including 669 patients with chronic heart failure[15] has demonstrated that adding trimetazidine to conventional therapy for heart failure is effective in reducing mortality and hospitalisations for heart failure. Several meta-analyses of randomised controlled trials have consistently confirmed the findings of the studies on the effect of trimetazidine in heart failure and have confirmed that trimetazidine improves cardiac function and exercise capacity, reduces mortality, cardiovascular events and hospitalisations in both ischaemic and non-ischaemic heart failure.[27]

Because of the strength of clinical evidence on its efficacy trimetazidine has been included in the guidelines for the treatment of heart failure with reduced ejection fraction to be used in patients with heart failure of ischaemic aetiology used as addon to an angiotensin-converting enzyme (ACE) inhibitor (or an angiotensin receptor blocker if ACE inhibitors are not tolerated), a beta-blocker and a mineralocorticoid receptor antagonist.

\section{Perhexiline}

Like trimetazidine, perhexiline had also been originally developed as an anti-anginal medication. This drug was initially marketed in the 1970s but its use declined due to the reports of serious adverse events, including hepatotoxicity and neurotoxicity. Subsequently, it has been found that the toxicity could be preventable with individualised dosing and dosage titration to steady state levels between 150 and $600 \mathrm{mg} / \mathrm{mL}$. A few small placebo-controlled clinical studies suggest that this compound may be effective in heart failure, but the evidence to support its use is at the present very weak.

An improvement in peak oxygen consumption was found following perhexiline treatment compared to no change in patients treated with a placebo.[28] In the same study perhexiline was found to improve left ejection fraction, symptoms, resting and peak stress myocardial function and skeletal mass energetics. Other clinical trials have shown an improvement in symptoms of heart failure in patients treated with perhexiline.[29-31] A recent study found that treatment improved cardiac energetics given the improvement in the phosphocreatine (PCr)/ATP ratio but no significant change in left ventricular function or BNP following perhexiline treatment.[29]. 


\section{Etomoxir}

Etomoxir is an inhibitor of CPT-1, similar to perhexiline. Animal studies suggested that etomoxir improved cardiac function in diabetic rats, and therefore the compound had been tested in humans.[32] However, in a randomized placebo-controlled trial etomoxir including 347 patients was stopped early due to unacceptably high liver transaminase levels. Given the safety concerns, the clinical development of etomoxir has been halted.

\section{Dichloroacetate}

Dichloroacetate is an inhibitor of pyruvate dehydrogenase kinase that increases mitochondrial pyruvate dehydrogenase and in turn glucose oxidation. Small studies have shown that dichloroacetate improves left ventricular function, also seen in animal models.[33] Subsequent clinical trials in patients with heart failure patients did not detect any significant benefit of dichloroacetate and its clinical development has been stopped.[34-35]

\section{Metabolic-like agents}

Other agents with metabolic effects on the myocardium increase high energy phosphate production without altering metabolism, but rather by facilitating electron transport on the surface of the mitochondrial cristae.

Elamipretide is an aromatic-cationic tetrapeptide that binds selectively to cardiolipin via electrostatic and hydrophobic interactions. This small molecule protects cardiolipin from oxidative stress and preserves the function of the respiratory electron chain. Elamipretide also prevents cardiolipin from converting cytochrome $\mathrm{c}$ into a peroxidase and protects its electron carrying function. Therefore, it partially stabilises and partially normalises the dysfunctional mitochondria of cardiomyocytes and other tissues including skeletal muscle. It partly increases the electron flux through cytochrome $\mathrm{C}$ and reduces electron leak as well as increasing the maximum rate of ATP synthesis and reducing the production of reactive oxygen species.[36] It has undergone early clinical trial evaluation in mitochondrial myopathies[37] and shows promise pre-clinically for CHF both by targeting the metabolically stressed heart but also by improving skeletal muscle function.[38,39] Elamipretide improved left ventricular systolic function and stroke volume without affecting heart rate nor blood pressure in a dog model of systolic heart failure. This drug is currently been tested in a phase II study in patients with acute heart failure.

Coenzyme Q10, also known as ubiquinone is an important component of electron transport on the mitochondrial cristae undergoing cyclical oxidation and reduction. Depletion of Coenzyme Q10 can slow mitochondrial respiration in the cardiomyocytes while its supplementation accelerate respiration. Nearly $50 \%$ of coenzyme Q10 comes from food while the remainder is synthesised through the mevalonate pathway. This pathway is blocked by statins that reduce coenzyme Q10 levels. Levels of coenzyme Q10 inversely correlate to the severity of the disease in patients with heart failure. And low levels of coenzyme Q10 had been associated with increased mortality. Coenzyme Q10 supplementation has been associated with improved left ventricular function in small size studies. More recently the Q-Symbio study tested the effect of coenzyme Q10 compared to placebo in 420 patients with heart failure and reported an improvement of symptoms and a trend towards a reduction in

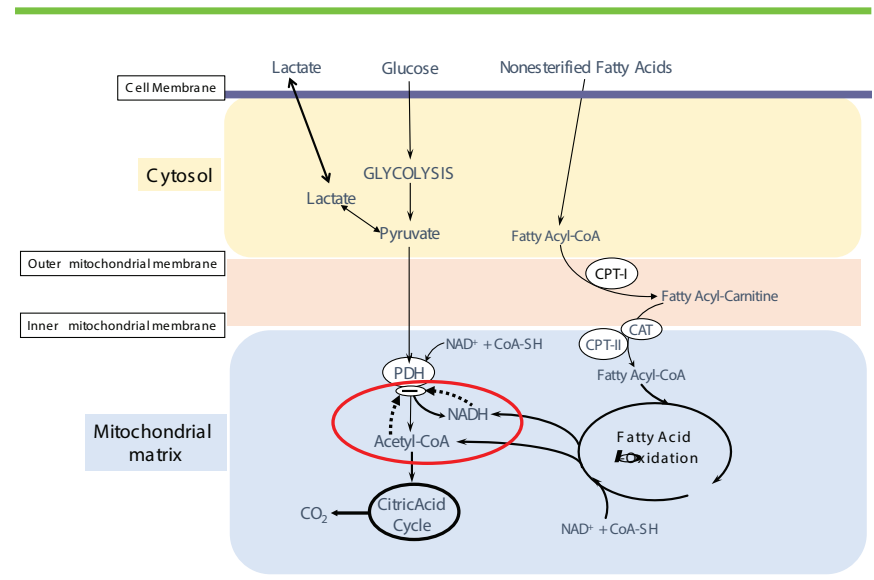

Figure 1. Metabolic pathways of glucose and non-esterified fatty acids in the cytosol and at the mitochondria level in cardiac myocytes.

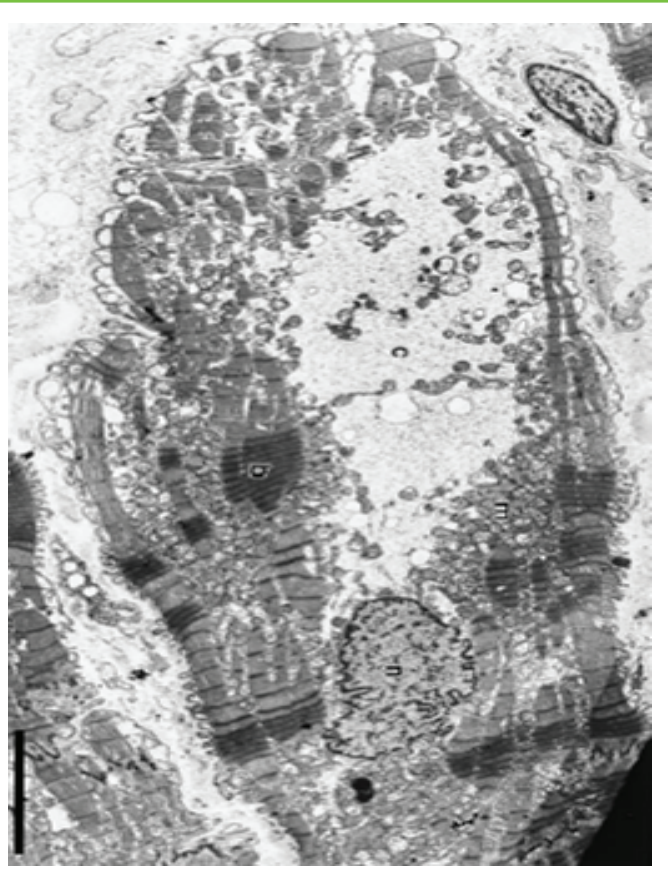

Figure 2. Electron microscope image of cardiac myocytes in failure with reduced ejection fraction showing reduced myofibrillar content, glycogen accumulation, small rounded mitochondria with reduced cristae, irregular nuclear envelopes

cardiovascular events. The efficacy of coenzyme Q10 for the treatment of heart failure will need more supportive data before it can be endorsed.

MitoQ is a mitochondrial targeted anti-oxidant where an ubiquinone-derivative, ubiquinol, is linked to the cation triphenylphosphonium (TPP+) by a 10-carbon alkyl chain. TPP+ is used to take advantage of the electrochemical gradient across the inner mitochondrial membrane and therefore, increases by several hundred fold the concentration of ubiquinol across the mitochondria. MitoQ can be administered orally and preclinical studies have shown efficacy in protecting against oxidative damage. Preclinical studies suggested a protective effect of MitoQ against anthracycline and cocaine toxicity. To date MitoQ has been tested in patients with Parkinson's disease and chronic hepatitis $C$ but not in heart failure. 


\section{Conclusions}

A maladaptive cardiac metabolism occurs early in patients with heart failure and impairs cardiac and skeletal muscular function. Modulation of cardiac metabolism with partial inhibition of free fatty acid oxidation has been shown to be beneficial in patients with heart failure. At the present, the bulk of evidence for this class of drugs comes from Trimetazidine. Newer compounds partially inhibiting free fatty acid oxidation or facilitating the electron transport on the mitochondrial cristae are in early phase of their clinical development.

\section{Declarations of interest}

The authors declare no conflict of interest.

\section{Acknowledgements}

The authors state that they abide by the authors' responsibilities and ethical publishing guidelines of the International Cardiovascular Forum Journal.[40]

\section{References}

1. Doehner W, Frenneaux M, Anker SD. Metabolic impairment in heart failure: the myocardial and systemic perspective. J Am Coll Cardiol. 2014;64:1388-1400.

2. Neubauer $\mathrm{S}$. The failing heart--an engine out of fuel. $\mathrm{N}$ Engl $\mathrm{J}$ Med. 2007;356:1140-1151.

3. Fillmore N, Mori J, Lopaschuk GD. Mitochondrial fatty acid oxidation alterations in heart failure, ischaemic heart disease and diabetic cardiomyopathy. Br J Pharmacol 2014;171:2080-90.

4. Gertz EW, Wisneski JA, Stanley WC, Neese RA. Myocardial substrate utilization during exercise in humans. Dual carbon-labeled carbohydrate isotope experiments. J Clin Invest. 1988;82:2017-2025.

5. Abozguia K, Shivu GN, Ahmed I, Phan TT, Frenneaux MP. The heart metabolism: pathophysiological aspects in ischaemia and heart failure. Curr Pharm Des. 2009;15:827-835.

6. Weber KT, Janicki JS. The metabolic demand and oxygen supply of the heart: physiologic and clinical considerations. Am J Cardiol. 1979;44:722-729

7. Lommi J, Koskinen P, Naveri H, Harkonen M, Kupari M. Heart failure ketosis. J Intern Med. 1997;242:231-238.

8. Lommi J, Kupari M, Koskinen P, Naveri H, Leinonen H, Pulkki K, Harkonen M. Blood ketone bodies in congestive heart failure. J Am Coll Cardiol. 1996;28:665-672.

9. Doenst T, Nguyen TD, Abel ED. Cardiac metabolism in heart failure: implications beyond ATP production. Circ Res. 2013;113:709-724.

10. Heusch G, Libby P, Gersh B, Yellon D, Bohm M, Lopaschuk G, Opie L. Cardiovascular remodelling in coronary artery disease and heart failure. Lancet. 2014;383:1933-1943.

11. Fragasso G Deranged Cardiac Metabolism And The Pathogenesis Of Heart Failure. Cardiac Failure Review, 2016;2[1]:8-13.

12. Lionetti V, Stanley WC, Recchia FA. Modulating fatty acid oxidation in heart failure. Cardiovasc Res. 2011;90:202-209.

13. Beadle RM, Williams LK, Kuehl M, Bowater S, Abozguia K, Leyva F, Yousef Z, Wagenmakers AJ, Thies F, Horowitz J, Frenneaux MP. Improvement in Cardiac Energetics by Perhexiline in Heart Failure Due to Dilated Cardiomyopathy. JACC Heart Fail. 2015. Mar;3(3):202-11. doi: 10.1016/j. jchf.2014.09.009.

14. Lee L, Campbell R, Scheuermann-Freestone M, Taylor R, Gunaruwan P, Williams L, Ashrafian H, Horowitz J, Fraser AG, Clarke K, Frenneaux M. Metabolic modulation with perhexiline in chronic heart failure: a randomized, controlled trial of short-term use of a novel treatment. Circulation. 2005;112:3280-3288.

15. Fragasso G, Rosano G, Baek SH, Sisakian H, Di Napoli P, Alberti L, Calor G, Kang SM, Sahakyan L, Sanosyan A, Vitale C, Marazzi G, Margonato A, Belardinelli R. Effect of partial fatty acid oxidation inhibition with trimetazidine on mortality and morbidity in heart failure: results from an international multicentre retrospective cohort study. Int J Cardiol. 2013;163:320-325.

16. Kantor PF, Lucien A, Kozak R, Lopaschuk GD. The antianginal drug trimetazidine shifts cardiac energy metabolism from fatty acid oxidation to glucose oxidation by inhibiting mitochondrial long-chain 3-ketoacyl coenzyme A thiolase. Circ Res. 2000;86:580-588

17. Vitale C, Wajngaten M, Sposato B, Gebara O, Rossini P, Fini M, Volterrani M, Rosano GM. Trimetazidine improves left ventricular function and quality of life in elderly patients with coronary artery disease. Eur Heart $\mathrm{J}$. 2004;25:1814-1821.
18. Khan M, Meduru S, Mostafa M, Khan S, Hideg K, Kuppusamy P. Trimetazidine, administered at the onset of reperfusion, ameliorates myocardial dysfunction and injury by activation of p38 mitogen-activated protein kinase and Akt signaling. J Pharmacol Exp Ther. 2010;333:421-429.

19. Brottier L, Barat JL, Combe C, Boussens B, Bonnet J, Bricaud H. Therapeutic value of a cardioprotective agent in patients with severe ischaemic cardiomyopathy. Eur Heart J. 1990;11:207-212.

20. Fragasso G, Piatti Md PM, Monti L, Palloshi A, Setola E, Puccetti P, Calori G, Lopaschuk GD, Margonato A. Short- and long-term beneficial effects of trimetazidine in patients with diabetes and ischemic cardiomyopathy. Am Heart J. 2003;146:E18.

21. Rosano GM, Vitale C, Sposato B, Mercuro G, Fini M. Trimetazidine improves left ventricular function in diabetic patients with coronary artery disease: a double-blind placebo-controlled study. Cardiovasc Diabetol. 2003;2:16.

22. Fragasso G, Palloshi A, Puccetti P, Silipigni C, Rossodivita A, Pala M, Calori G, Alfieri O, Margonato A. A randomized clinical trial of trimetazidine, a partial free fatty acid oxidation inhibitor, in patients with heart failure. J Am Coll Cardiol. 2006;48:992-998.

23. Di Napoli P, Di Giovanni P, Gaeta MA, D’Apolito G, Barsotti A. Beneficia effects of trimetazidine treatment on exercise tolerance and B-type natriuretic peptide and troponin $\mathrm{T}$ plasma levels in patients with stable ischemic cardiomyopathy. Am Heart J. 2007;154:602 e601-605.

24. Di Napoli P, Taccardi AA, Barsotti A. Long term cardioprotective action of trimetazidine and potential effect on the inflammatory process in patients with ischaemic dilated cardiomyopathy. Heart. 2005;91:161-165.

25. Belardinelli R, Purcaro A. Effects of trimetazidine on the contractile response of chronically dysfunctional myocardium to low-dose dobutamine in ischaemic cardiomyopathy. Eur Heart J. 2001;22:2164-2170.

26. Belardinelli R, Lacalaprice F, Faccenda E, Volpe L. Trimetazidine potentiates the effects of exercise training in patients with ischemic cardiomyopathy referred for cardiac rehabilitation. Eur $\mathrm{J}$ Cardiovasc Prev Rehabil. 2008;15:533-540.

27. Zhang L, Lu Y, Jiang H, Zhang L, Sun A, Zou Y, et al., Additional use of trimetazidine in patients with chronic heart failure: a meta-analysis, J. Am. Coll. Cardiol. 2012; 59: 913-922.

28. Lee L., Campbell R., Scheuermann-Freestone M., Taylor R., Gunaruwan P., Williams L., Ashrafian H., Horowitz J., Fraser A.G., Clarke K., et al. Metabolic modulation with perhexiline in chronic heart failure a randomized, controlled trial of short-term use of a novel treatment. Circulation. 2005;112:3280-3288.36.

29. Beadle R.M., Williams L.K., Kuehl M., Bowater S., Abozguia K., Leyva F. Yousef Z., Wagenmakers A.J., Thies F., Horowitz J., et al. Improvement in cardiac energetics by perhexiline in heart failure due to dilated cardiomyopathy. JACC: Heart Fail. 2015;3:202-211.

30. Abozguia K., Elliott P., McKenna W., Phan T.T., Nallur-Shivu G., Ahmed I., Maher A.R., Kaur K., Taylor J., Henning A., et al. Metabolic modulator perhexiline corrects energy deficiency and improves exercise capacity in symptomatic hypertrophic cardiomyopathy. Circulation. 2010;122:1562-1569.

31. Phan T.T., Shivu G.N., Choudhury A., Khalid A., Davies C., Naidoo U., Ahmed I., Yousef Z., Horowitz J., Frenneaux M. Multi-centre experience on the use of perhexiline in chronic heart failure and refractory angina: Old drug, new hope. Eur. J. Heart Fail. 2009;11:881-886.38.

32. Schmitz F.J., Rosen P., Reinauer H. Improvement of myocardial function and metabolism in diabetic rats by the carnitine palmitoyl transferase inhibitor Etomoxir. Horm. Metab. Res. 1995;27:515-522.

33. Kato T., Niizuma S., Inuzuka Y., Kawashima T., Okuda J., Tamaki Y., Iwanaga Y., Narazaki M., Matsuda T., Soga T., et al. Analysis of metabolic remodeling in compensated left ventricular hypertrophy and heart failure. Circ. Heart Fail. 2010;3:420-430.

34. Wilson J.R., Mancini D.M., Ferraro N., Egler J. Effect of dichloroacetate on the exercise performance of patients with heart failure. J. Am. Coll. Cardiol. $1988 ; 12: 1464-1469$

35. Lewis J.F., DaCosta M., Wargowich T., Stacpoole P. Effects of dichloroacetate in patients with congestive heart failure. Clin. Cardiol. 1998;21:888-892.

36. Birk AV, Chao WM, Bracken C, Warren JD, Szeto HH. Targeting mitochondrial cardiolipin and the cytochrome c/cardiolipin complex to promote electron transport and optimize mitochondrial ATP synthesis. $\mathrm{Br} \mathrm{J}$ Pharmacol. 2014;171:2017-2028.

37. Karaa A, Haas R, Goldstein A, Vockley J, Weaver WD, Cohen BH. Randomized dose-escalation trial of elamipretide in adults with primary mitochondrial myopathy. Neurology. 2018 Apr 3;90(14):e1212-e1221. doi: 10.1212/WNL.0000000000005255. Epub 2018 Mar 2. Erratum in: Neurology. 2018 Apr 3;90(14 ):669. Neurology. 2018 Nov 20;91(21):986.

38. Sabbah HN, Gupta RC, Singh-Gupta V, Zhang K, Lanfear DE. Abnormalities of Mitochondrial Dynamics in the Failing Heart: Normalization Following Long-Term Therapy with Elamipretide. Cardiovasc Drugs Ther. 2018 Aug;32(4):319-328. doi: 10.1007/s10557-018-6805-y.

39. Sabbah HN, Gupta RC, Singh-Gupta V, Zhang K. Effects of elamipretide on skeletal muscle in dogs with experimentally induced heart failure. ESC Heart Fail. 2019 Jan 28. doi: 10.1002/ehf2.12408

40. Shewan LG, Coats AJS, Henein MY. Authors' Responsibilities and Ethica Publishing. International Cardiovascular Forum Journal 2018;13:3-4, DOI: 10.17987/icfj.v13i0.525" 